\title{
Effective Spectrum Sensing By Weighted Data Fusion In Full Duplex Cognitive Radio Networks
}

\author{
Qiming Yu*, Hu Jin*, Yifan Liu* and Wenqing Zheng* \\ ${ }^{\star}$ Electronic Engineering Institute, Hefei 230000, China
}

Keywords: Full duplex, Cognitive radio, LAT protocol, Data fusion, Convex optimization

\begin{abstract}
As the full duplex (FD) technique becoming more sophisticated, a novel cognitive radio network (CRN) protocol called " listen-and-talk” (LAT) was proposed. Based on self-interference cancellation (SIC) technique, LAT protocol can promote the utilization of spectrum resources with the cost of the performance of spectrum sensing. Several researchers made efforts to overcome this shortcoming. In this paper, a more effective cooperative spectrum sensing strategy based on weighted data fusion was proposed. Convex optimization is used to solve the math model Simulation results show that the method of weighted data fusion (WDF) highly improves the performance of spectrum sensing in FDCRN, also outperforms the previous studies.
\end{abstract}

\section{Introduction}

The traditional cognitive radio needs to divide the time slot into the sensing period and the sending period. The data transmission of secondary users (SUs) can be carried out in the sending period only if primary users(PUs) are not detected in the sensing period. This model is called LBT (Listenbefore -talk) protocol. As the emergence of the primary users is random, LBT protocol may not guarantee to avoid data conflicting, the division of time slot also caused a certain waste of resources.

In 2011, some scholars first proposed the concept of fullduplex cognitive radio ${ }^{[1,2]}$, while the co-time co-frequency full-duplex technology makes the in-band simultaneously two-way communication into reality ${ }^{[3-5]}$. FDCRNs combine the two technology, and make the spectrum utilized more efficiently. In 2015, the principle of hardware and software for FDCRN was systematically stated by Liao.Y et al. And FDCRN are widely recognized to be a promising new communication technology for wide range of application prospects were proposed ${ }^{[6]}$. LAT protocol,enable the CRN transmitting and sensing simultaneously, is the key to FDCRN. In Ref.[7], the detection probability and false alarm probability under LAT protocol are deduced. Based on this, the performance of LAT and LBT in detection capability and sub-user throughput were compared : 1) spectrum sensing performance of LAT can not match the performance of LBT. 2) In LAT, the throughput is limited by the SUs transmit power. In Ref.[8], cooperative and non-cooperative scenes of energy detection were analyzed. Also the throughput of SUs in LAT was optimized by particle swarm optimization algorithm, which is better than the results get in Ref.[7]. However, there is no optimization strategy for spectrum sensing performance of LAT protocol at present.

In LBT, the method of weighted data fusion were proved efficient to improve the spectrum sensing capability. In Ref.[9], two typical weighting methods were presented: 1) equal gain weighting 2) based on the weighting of the signalto-noise ratio. However, neither of these methods can adapt to LAT protocol for these two reasons:1) Equal gain weighting can not eliminate the influence of user self-interference, so it barely get the optimization effect. 2) Signal-to-noise weighting method in the LAT is difficult to conduct because the receivers get signals from both PU and SU1, this make the coefficient is hard to get.

Based on the analysis above, this paper focus on the cooperative spectrum sensing based on data fusion weighting in LAT. In the secondary users network, a small amount of prior information such as geographical location is used to define the weight of the current time slot for all the sensing users according to the usage of the current spectrum. When solving the mathematical model, convex optimization is used. To solve the optimal weight, the method of sequential programming is used to deal with the non-convex nonlinear problem. After get the optimal weighting coefficients, we choose some reliable SUs and make reliable decisions at the data fusion center(DFC). Simulations show that weighted data fusion method significantly improves the sensing ability, and has greatly overcome the negative impact brought from the full-duplex system.

\section{System model}

The research scenario in this paper is correspondent to Ref.[7]: The full-duplex cognitive radio network is consisted of a PU pair and SUs. Each user is equipped with two antennas. SUs have the ability to suppress self-interference. In a band, the primary users randomly appears, once the primary users turn on, all secondary users must stop working. When the primary users shut off in this band, SUs compete for a working user SU1, the rest of the users SUi working as collaborative sensing users. That is, the band can only have one working secondary user. SU1 working with simultaneously sending 
and sensing. Energy detection was adopted to sensing the spectrum for SUi.

Based on the theory of statistical testing, we select test statistic as:

$$
T_{i}=\frac{1}{M} \sum_{k=1}^{M}\left|r_{i}(k)\right|^{2}
$$

where $M$ denotes the number of samples, and $r_{i}(k)$ is the received signal of the $n^{\text {th }}$ sample for the $i^{\text {th }}$ secondary user SUi. Let $f_{S}$ represent the sampling frequency in sensing process, and for sensing duration $t$, we have $M \propto f_{S} t$.

$$
\begin{array}{r}
P_{f}^{0}\left(P_{m}^{0}\right)=Q\left(Q^{-1}\left(1-P_{m}^{0}\right)\left(1+\gamma_{p}\right)+\gamma_{p} \sqrt{M}\right) \\
\text { 3) } \quad P_{f}^{1}\left(P_{m}^{1}\right)=Q\left(Q^{-1}\left(1-P_{m}^{1}\right)\left(\frac{1+\gamma_{p}+\gamma_{s}}{1+\gamma_{s}}\right)+\frac{\gamma_{p}}{1+\gamma_{s}} \sqrt{M}\right)
\end{array}
$$

\begin{tabular}{c|c|c|c|c|c}
\hline $\begin{array}{c}\text { hypo } \\
\text { thesi } \\
\mathrm{s}\end{array}$ & $E\left\lfloor T \mid H_{i j}\right\rfloor$ & $\operatorname{Var}\left\lfloor T \mid H_{i j}\right\rfloor$ & $\begin{array}{c}\text { hypo } \\
\text { thesi } \\
\mathrm{s}\end{array}$ & $E\left\lfloor T \mid H_{i j}\right\rfloor$ & $\operatorname{Var}\left\lfloor T \mid H_{i j}\right\rfloor$ \\
\hline$H_{00}$ & $\sigma_{n}^{2}$ & $\frac{\sigma_{n}^{4}}{M}$ & $H_{10}$ & $\left(1+\gamma_{p}\right) \sigma_{n}^{2}$ & $\frac{\left(1+\gamma_{p}\right)^{2} \sigma_{n}^{4}}{M}$ \\
\hline$H_{01}$ & $\left(1+\gamma_{s}\right) \sigma_{n}^{2}$ & $\frac{\left(1+\gamma_{s}\right)^{2} \sigma_{n}^{4}}{M}$ & $H_{11}$ & $\left(1+\gamma_{s}+\gamma_{p}\right) \sigma_{n}^{2}$ & $\frac{\left(1+\gamma_{s}+\gamma_{p}\right)^{2} \sigma_{n}^{4}}{M}$ \\
\hline
\end{tabular}

Table I The mean and variance of the test statistic under four hypotheses

In LAT, collaborative sensing users SUi may receive signals from both PU and SU1, which makes the detection hypothesis more complex than the binary hypothesis in LBT. We present the hypothesis as follows:

$$
r= \begin{cases}n & H_{00} \\ h_{p} p+n & H_{01} \\ h_{s} s+n & H_{10} \\ h_{p} p+h_{s} s+n & H_{11}\end{cases}
$$

where $H_{i j}$ denotes different states of FDCRN, $H_{00}$ represents both PU and SU1 keep silence, $H_{01}$ represents PU is busy and SU1 is silent, $H_{10}$ represents PU is idle and SU1 is active, $H_{11}$ represents PU and SU1 are both active. $p$ denotes the signal from $\mathrm{PU}, h_{p}$ denotes the channel gain from PU to SUi, $s$ denotes the signal from SU1, $h_{S}$ denotes the channel gain from SU1 to SUi. $n$ denotes the AWGN.

According to the AWGN hypothesis and the central limit theorem in detection hypothesis (2), it can be deduced that the statistical detection $T$ can be approximately treated as the Gaussian distribution under each hypothesis when $M \geq 100$. $T \sim N\left(\mu_{i j}, \sigma_{i j}^{2}\right)$. The mean and variance under each hypothesis is shown in Table I. Where $\gamma_{p}$ denotes the PUsignal-to-noise ratio, $\gamma_{S}$ denotes the SU1-signal-to-noise ratio,and $\sigma_{n}^{2}$ denotes the variance of Gaussian white noise.

In Ref.[7], the discrete-time Markov model is used to derive the system's miss detection probability and false alarm probability, shows in Table II.

According to Table II (3)(4) can be easily derived:

\begin{tabular}{c|c|c}
\hline & $P_{f}$ & $P_{m}$ \\
\hline $\begin{array}{c}\text { SU is } \\
\text { inactive }\end{array}$ & $P_{f}^{0}=Q\left(\left(\frac{\varepsilon_{0}}{\sigma_{n}^{2}}-1\right) \sqrt{M}\right)$ & $P_{m}^{0}=1-Q\left(\left(\frac{\varepsilon_{0}}{\left(1+\gamma_{p}\right) \sigma_{n}^{2}}-1\right) \sqrt{M}\right)$ \\
in is is & $P_{f}^{1}=Q\left(\left(\frac{\varepsilon_{1}}{\left(1+\gamma_{s}\right) \sigma_{n}^{2}}-1\right) \sqrt{M}\right)$ & $P_{m}^{1}=1-Q\left(\left(\frac{\varepsilon_{1}}{\left(1+\gamma_{p}+\gamma_{s}\right) \sigma_{n}^{2}}-1\right) \sqrt{M}\right)$ \\
LAT & $P_{f}=\frac{P_{f}^{1}}{1-P_{f}^{0}+P_{f}^{1}}$ & $P_{m}=\frac{P_{m}^{0}}{1-P_{m}^{1}+P_{m}^{0}}$ \\
\hline
\end{tabular}

Table II The false alarm probability and miss detection probability under four hypotheses under LAT protocol, which is shown in Figure 1. Fig. 1 shows that when the intensity of SU1' s power could undermine the detection probability of PU in LAT protocol. Which could result in the data collision, and data collision is very harmful to the communication in cognitive radios.

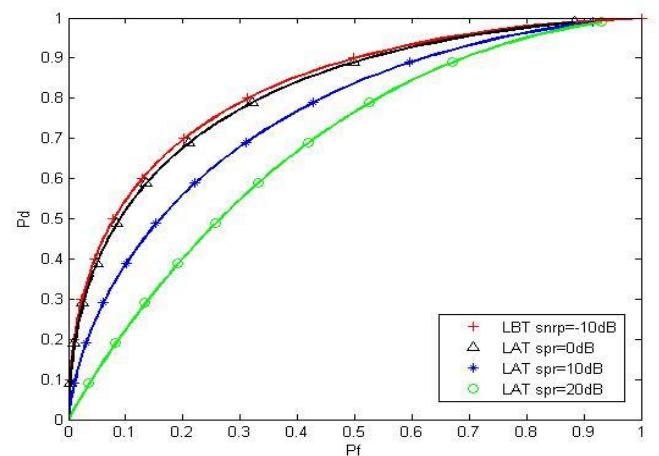

Fig. 1 detection performance comparison between LAT and LBT

\section{Weighted Data Fusion Algorithm}

Data fusion means that each sensing users SUi make the decision locally based on the information received. Then each SUi sends the decision (0/1) to DFC, at DFC the decisions are fused by different weight and get a more reliable decision. This algorithm can eliminate the unreliable decisions made by
Once $\gamma_{p}$ and $\gamma_{s}$ is determined, we can plot the ROC curve 
the sensing nodes which is close to SU1 with high SU-SNR, and raise the reliability.

The process is detailed in Fig 2.

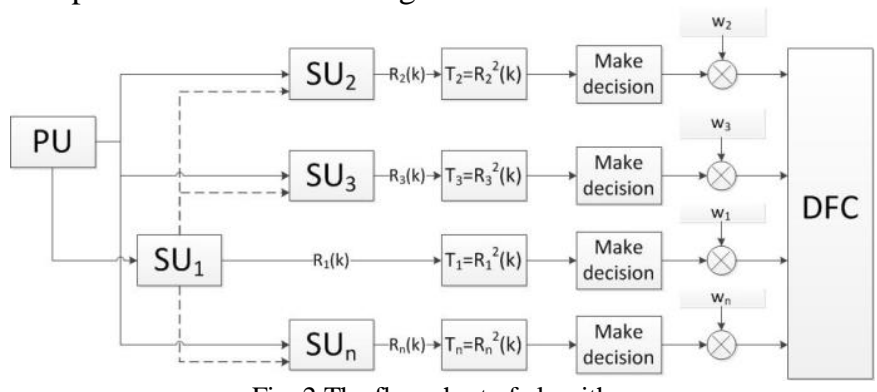

Fig. 2 The flow chart of algorithm

\begin{tabular}{ccc}
\hline & AND & OR \\
\hline $\begin{array}{c}\text { false alarm } \\
\text { probability } P_{f, s} \\
\text { detection } \\
\text { probability } P_{d, s}\end{array}$ & $P_{f, s}=\prod_{i=1}^{N} P_{f, i}$ & $P_{f, s}=1-\prod_{i=1}^{N}\left(1-P_{f, i}\right)$ \\
\hline Table III Common Criteria for Decision Fusion
\end{tabular}

The math model can be established as:

$$
\begin{array}{ll}
\min & P_{f, s}=\sum_{i=1}^{N} \omega_{i} P_{f, i} \\
\text { s.t. } & \boldsymbol{I}^{\boldsymbol{T}} \boldsymbol{\omega}=1 \\
& \boldsymbol{\omega} \succ 0
\end{array}
$$

In (5) is the false alarm probability of SUi, and can be calculated by Table II and (3)(4). It can be easily proved that the objective function and constraint conditions are convex. So this math model can be solved by CVX toolbox.

\section{Simulation}

\begin{tabular}{|c|c|}
\hline \multicolumn{1}{|c|}{ Table IV Simulation Parameters } \\
\hline $\begin{array}{c}\text { Parameters } \\
\text { The sampling frequency }\left(f_{s}\right)\end{array}$ & $1 \mathrm{MHz}$ \\
\hline $\begin{array}{c}\text { The duration of sensing time } \\
(t)\end{array}$ & $0.2 \mathrm{~ms}$ \\
\hline $\begin{array}{c}\text { The number of samples in LAT } \\
(M)\end{array}$ & 200 \\
\hline $\begin{array}{c}\text { The relative noise variance } \\
\left(\sigma_{n}^{2}\right)\end{array}$ & 1 \\
\hline PU-SNR $\left(\gamma_{p}\right)$ & $(-13 \mathrm{~dB},-6 \mathrm{~dB})$ \\
\hline SU-SNR $\left(\gamma_{s}\right)$ & $(-20 \mathrm{~dB}, 20 \mathrm{~dB})$ \\
\hline SIC factor of LAT $(\xi)$ & 0.15 \\
\hline
\end{tabular}

The simulation background takes the 802.22WRAN model ${ }^{[10]}$. By using the Monte Carlo method, the secondary users randomly appear in the circle with the PU is located in the origin point. The signal propagation takes the near-ground communication model, that is, the power density of the received signal decrease in proportional to the transmission distance. According to the current engineering product the SIC factor can be achieved approximately as 0.15 .

Analysis of the simulation:

1) Figure 3: In this simulation, the number of SUs is five. The five curves are the ROC curves from all these five SUs. It shows that different SUs at different location has different ROC curve, that means the interference from the SU1 signal varies from the distance between SU1 and sensing user SUi. The longer distance means the more signal attenuation, so the best sensing usually located far from SU1 and close to PU, this help to make a more accurate decision. In traditional data fusion algorithms, no matter AND $\backslash \mathrm{OR} \backslash \mathrm{k} / \mathrm{N}$, they get the mean of different curves in different ways to make the last decision more accurate, but the mean of different sensing users always involves in some "bad" decision, inevitably. The algorithm proposed in this paper can achieve the detection probability which the best sensing user achieved and get rid of all "bad" decisions. It is shown clearly in Figure 3.

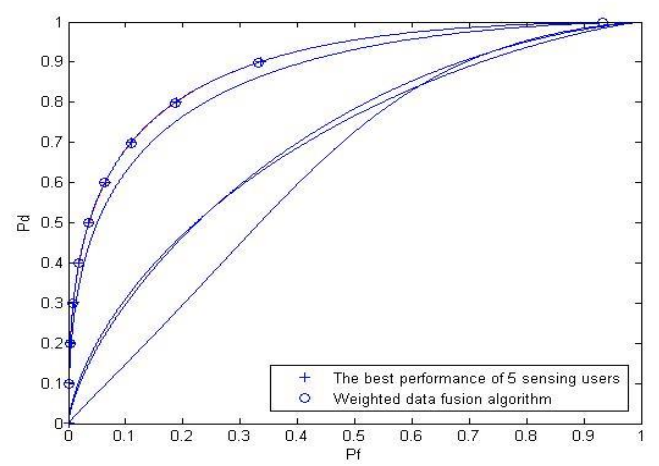

Figure 3. N=5 Weighted Data Fusion

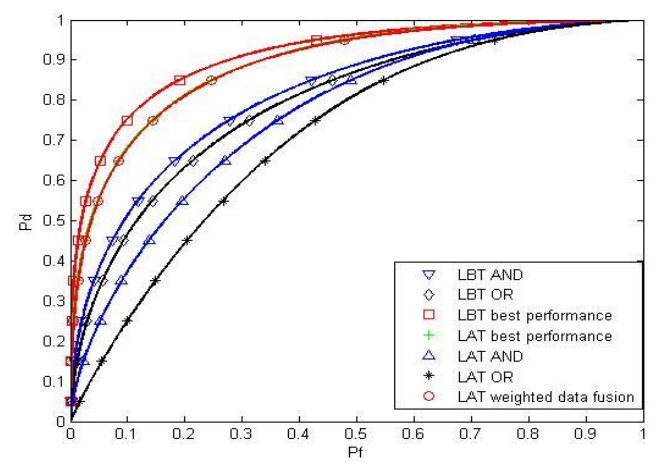

Figure 4. N=20 Comparison between LAT and LBT under ANDIORIWDF

2) Figure 4: This simulation shows the comparison between weighted data fusion algorithm and ANDIOR criteria in both LAT and LBT protocol. The number of this simulation involves 20 secondary users. We can see that in both LAT and LBT protocol, the weighted data fusion algorithm can get the best sensing user' $\mathrm{s}$ detection probability, and outperform the AND $\backslash O R$ criteria. Then AND criteria is always have a better performance than OR criteria, this is because OR criteria have a higher false alarm probability. In CRN, miss detection probability is more important than false alarm 
probability, for miss detection probability could cause data collision and false alarm probability could only cause spectrum waste. So the algorithm proposed in this paper obviously perform better than traditional criteria.

3) Figure 4: In Fig.1 we show that the detection performance of LBT is usually better than LAT. This simulation also shows that the weighted data fusion algorithm can greatly reduce the difference performance between LAT and LBT, which in ANDIOR the difference is remarkable. Weighted data fusion algorithm can make LAT greatly approach the performance of LBT.

\section{Conclusion}

In order to solve the problem of limited detection performance in the full-duplex cognitive radio LAT protocol, this paper proposes a weighted data fusion algorithm based on cooperative energy detection. The mathematical model is solved by convex optimization. The simulation results show that the algorithm in this paper have better performance than the AND and OR criteria. Decision fusion is weighted to obtain the best node performance in the sub-user network The method proposed has greatly overcome the shortcomings brought by the full-duplex system, and LAT system performance has been further improved.

\section{References}

[1].Cheng, Wenchi, X. Zhang, and H. Zhang. "Full Duplex Wireless Communications for Cognitive Radio Networks." Mathematics (2011).

[2].Cheng, Wenchi, X. Zhang, and H. Zhang. "Imperfect full duplex spectrum sensing in cognitive radio networks." IEEE, 2011:1-6

[3].Choi, Jung Il, et al. "Achieving single channel, full duplex wireless communication." International Conference on Mobile Computing and Networking, MOBICOM 2010, Chicago, Illinois, Usa, September DBLP, 2010:1-12.

[4].Everett, Evan, A. Sahai, and A. Sabharwal. "Passive SelfInterference Suppression for Full-Duplex Infrastructure Nodes." IEEE Transactions on Wireless Communications 13.2(2013):680-694.

[5].Duarte, M, and A. Sabharwal. "Full-duplex wireless communications using off-the-shelf radios: Feasibility and first results." Signals, Systems and Computers IEEE, 2010:1558-1562.

[6].Liao, Yun, et al. "Full duplex cognitive radio: a new design paradigm for enhancing spectrum usage." IEEE Communications Magazine53.5(2015):138-145.

[7].Liao, Yun, et al. "Listen-and-talk: Full-duplex cognitive radio networks."IEEE Global Communications Conference IEEE, 2014:3068-3073.

[8].Liao, Y., et al. "Cooperative spectrum sensing for fullduplex cognitive radio networks." IEEE International Conference on Communication Systems IEEE, 2014:56-60.
[9].Ma, Jun, G. Zhao, and Y. Li. "Soft Combination and Detection for Cooperative Spectrum Sensing in Cognitive Radio Networks." IEEE Transactions on Wireless Communications 7.11(2008):4502-4507.

[10].IEEE, By. "802.22 Wireless RAN. Functional requirements for the 802.22 WRAN." (2010). 